\title{
Mapping the quality of basic and comprehensive emergency obstetric care services in Haiti.
}

Adeyinka E Adegbosin ${ }^{1}$, Jan Marnken ${ }^{2}$, Jing Sun ${ }^{1 *}$

${ }^{1}$ School of Medicine, Griffith University, Gold Coast, Queensland

${ }^{2}$ School of Environment and Science, Griffith University, Gold Coast, Queensland

*Corresponding Author: email - J.sun@griffith.edu.au, Tel - 0756780924, Address - Level 8, G40,

Griffith University School of Medicine, Parkland Drive, Gold Coast, 4222,

\section{Mapping the quality of basic and comprehensive emergency obstetric care services in Haiti.}

\section{Abstract \\ Objective}

To investigate geographical inequalities and changes in the quality of emergency obstetric care services available in Haiti over time.

\section{Methods}

We utilised data from the Service Provision Assessment (SPA) survey of all health facilities in Haiti in 2013 and 2017. We developed a quality index for Basic and Comprehensive Emergency Obstetric Care (BEmOC and CEmOC) based on the items in the signal functions of emergency obstetric care framework, using a structure, process and outcome framework. We measured the quality index of all facilities in 2013 and 2017. We also assessed geographical trends and changes in quality between 2013 and 2017 using geospatial analysis.

\section{Result}

Our analysis showed that basic structure items such as connection to electricity grid, manual vacuum extractors, vacuum aspirators and dilation and curettage kits were widely unavailable at health care facilities. There was a significant improvement in indicators of structure ( $p<$ $0.001)$ and BEmOC ( $p=0.03)$ in primary facilities, however there was no significant change 
in quality of CEmOC in primary facilities $(p=0.18)$. Similarly, there was no significant change in any of the structure or process indicators at secondary care facilities structure items

\section{Conclusion}

The availability of basic emergency obstetric care at several Haitian facilities remains poor, however there was significant improvement at primary care facilities, with little to no change in overall quality at secondary health facilities.

\section{Introduction}

Despite remarkable improvement in obstetric care coverage in Low-and Middle-Income Countries (LMIC), there remains an unacceptable high number of pregnancy related deaths in these countries (1). Studies have shown that the increase in the number of health-care facility births in low-income countries, may not sufficiently translate into reduction in maternal or newborn mortality due to the poor quality of care $(2,3)$.

Over the past few decades, efforts to monitor and evaluate Maternal, Newborn and Child Health (MNCH) care services in LMIC has been directed towards evaluating service coverage and utilisation, with very few studies exploring service quality (2). The few studies conducted have consistently shown that despite the increase in $\mathrm{MNCH}$ intervention coverage and increase in the availability of institutional care coverage, many avoidable deaths still occur during the perinatal period, many of which is due to poor quality of services $(1,4)$.

Studies have shown that timely recognition of obstetric complications and effective treatment of these complications is critical for reducing maternal mortality (5-7). Significant work has been done over the past three decades to develop reliable and internationally comparable indicators for measuring progress in obstetric care service coverage and quality. One of such 
important works, is the "guideline for monitoring availability and use of obstetric services", published by UNICEF, WHO and UNFPA in 1997 (8). This guideline contained an organising framework, which encompasses interventions and services required for the management of common pregnancy and child birth related complications $(8,9)$. These interventions and services were termed the signal functions of Basic Emergency Obstetric Care (BEmOC) and Comprehensive Emergency Obstetric Care CEmOC (9). The signal functions of BEmOC and $\mathrm{CEmOC}$ are an easier approach for measuring progress towards reduction of maternal mortality, when compared to other indicators such as maternal mortality rates or ratio $(8,9)$.

Significant gaps remain in the existing body of evidence on the quality of obstetric care services in LMIC. Many of the previous studies have focused on exploring a broad range of issues such as: cross-sectional analysis of quality and service readiness in LMIC (3); linking quality of $\mathrm{MNCH}$ services to the level of utilisation of delivery care $(4,5)$; developing summary indices for measuring quality of care (12); investigating association between service quality and client satisfaction (13).

Furthermore, previous studies have also explored the coverage and access to BEmOC and CEmOC in different countries: a recent systematic review identified twenty-seven of such studies (14). Bosomprah et al. conducted an analysis of the spatial distribution of emergency obstetric and newborn care in Ghana, and compared existing coverage with the minimum acceptable coverage level, showing that the country was below acceptable international standards (15). Gabrysch et al. explored geographical access to emergency obstetric care in Zambia (16). Hirosê et al. conducted a spatial modelling of factors contributing to delays in reaching an emergency obstetric facility in Afghanistan (17). A number of studies have also conducted a snap-shot analysis of the quality of emergency obstetric care at a given point in time in varioús countries $(13,18,19)$.

We identified 24 items that are associated with the signal function of BEmOC and CEmOC $(10,11)$. We stratified health facilities into primary facilities (non-hospitals) and secondary facilities (hospitals). We grouped these items into structures and processes based on a proxy of the Donabedian dimensions (12). This framework was then used to explore national and sub-national change in obstetric care quality in Haiti between 2013 and 2017. While our approach may serve to provide some context regarding the quality of obstetric care in Haiti and geographical differences. However, Donabedian's work on the seven attributes that 
defines health care highlights other parameters such as accessibility, cost and benefit, conformity to social preferences, provider-client relationship amongst other factors (21). Some of these more nuanced measures of quality may require a broader set of tools to assess them. Notably, there is no study till date that explored geographical change over time in emergency obstetric care quality in any given country. Our objective in this study, is to fill this gap in literature, by creating an index based on signal functions of BEmOC and CEmOC and using geospatial approach to monitor geographical disparity and changes in the quality of emergency obstetric care services in Haiti over time.

\section{Method}

\section{Data source}

We used two waves (2013 and 2017) of the Haiti Service Provision Assessment (SPA) survey, to explore change in the quality of emergency obstetric care over time. The SPA is a nationally representative facility-based cross-sectional survey, which is conducted periodically by the Demographic Health Survey (DHS) program. The survey includes data on healthcare service quality, which is derived through facility audits, healthcare worker survey, client observation and interviews. Further details on the SPA survey are available elsewhere (13). The facility coordinates were derived from the SPA GPS dataset, they represent the true location of the facility and are not displaced. The DHS program however states that the coordinates may not align precisely with the GIS data due to slight variations in shapefiles or generalisation of administrative boundaries. We derived the Haitian administrative boundary coordinates data from the Global Administrative Boundary Database (https://gadm.org/data.html).

\section{Settings \& survey design}


The study sample is made up of all public and private health facilities in Haiti in 2013 and 2017. This sample was drawn from a cross-sectional survey of all facilities conducted by the Demographic and Health Survey (DHS) programme in 2013 and 2017, respectively. The SPA is designed to address four broad groups of questions as follows:

(1). What is the availability of different health services in a country? Specifically, what proportions of the different facility types offer specific health services?

(2). To what extent are facilities prepared to provide health services? Do facilities have the necessary infrastructure, resources, and support systems available?

(3). To what extent does the service delivery process follow generally accepted standards of care? Does the process follow in service delivery meet standards of acceptable quality and content?

(4). Are clients and service providers satisfied with the service delivery environment?

We selected Haiti for this study, due to readily available national facility data over two time periods (2013 and 2017). In addition, Haitian facilities provided an ideal setting for our study due to a number of contextual reasons. Firstly, Haiti has one of the highest maternal mortality levels globally and the highest in the western hemisphere with an estimate of 359 deaths for every 100,000 births (23). Secondly, the Haitian health setting provides insight into health system resilience and recovery from disaster. An earthquake in January 2010 significantly strained the Haitian health system, with several lives lost and 50 health facilities destroyed $(24,25)$. Notable health gains have been recorded over the past decade since the earthquake (26), it is important to assess these gains, and to explore changes in health service quality.

\section{Statistical analysis \& Measures \\ Facility characteristics}

A deseriptive analysis was conducted, exploring facility chrematistics such as whether a facility was located in an urban or rural area, facility level of care, management type, availability of normal delivery and laboratory services.

\section{Facility quality index}

A quality index was computed for facilities that performed normal delivery and newborn care in 2013 and 2017 respectively. We created a 24-item index comprising of structure and processes based on the signal functions of $\mathrm{BEmOC}$ and $\mathrm{CEmOC}$ services. The signal function 
indicators are standardised and includes processes that are routinely carried out in facilities providing obstetric services. The survey also collected process data over a 3-month period, during which many of the routine processes would have being performed.

In addition, the structure indicator included in our analysis are linked to the process indicator, such that the structure indicators explores whether a facility has the basic equipment required for the routine processes comprising the signal functions of BEmOC and CEmOC. For example, a manual vacuum extractor is often used for assisted vaginal delivery: which is part of the BEmOC. Similarly, suture material with needles may be required for both assisted vaginal delivery, if episiotomy was provided (BEmOC) as well as for Caesarean section (CEmOC). Vacuum aspirator or dilation and curettage (D\&C) kit will be necessary for the removal of retained products. As there is no consensus on the structure indicator items for obstetric facilities, we relied on evidence from previous literature to determine the composition of items that were included.

Furthermore, some of the indicators are also mutually exclusive, if a facility has performed one process indicator, it also suggests that delivery care has taken place during this time frame.

The availability of each indicator of structure was determined by both provider responses and verification by the interviewer as to whether an item was observed or not. The availability of each process indicators was determined from the response given by providers to questions regarding whether a service has been performed over the past 3 months (See Table 2).

We stratified by facility types into primary or secondary. Primary facilities are health centers, while secondary facilites are hospitals. Theoretically, primary care facilities in many LMICs are expected to provide BEmOC and promptly identify complications and refer to higher level facilities. This however may not be the reality in practice in many LMICs settings, wherein complications may not be identified on time, or providers may choose not to refer complicated cases. Indeed, some authors have now called for a service redesign, such that deliveries only take place in facilities with the highest quality (7). On the basis of these realities, we also assessed CEmOC in primary care facilities.

\section{Composite measures}

Two indices were computed using a simple additive calculation analysis as follows: 
The national quality score is an aggregative measure for each indicator. Each facility was allocated a score of 0 for each indicator of structure that was not observed or for a process indicator that has not been performed over the past 3 months. A score of 1 is allocated to each observed structure indicator or process indicator performed in the past 3 months. We calculated a quality score for all facilities in Haiti, by aggregating the percentage number of facilities where an indicator is observed, or a process was performed in the last 3 months. A

\section{Mean facility quality score}

The mean quality score is a facility-level composite score, this was required for sub-national geographical comparison. We calculated a score for each Haitian facility by computing a mean score of all observed structure or process indicator at each facility

\section{Statistical test}

We calculated percentage point difference in the structure and process indicators between 2013 and 2017 for facilities that have conducted normal delivery and newborn care during this period. A Wilcoxon Signed rank test was conducted to assess for significant difference in the indicators of structure, BEmOC and CEmOC processes respectively (see Table 3).

\section{Geospatial analysis}

Using the 2013 and 2017 survey data, we computed a mean quality score for each facility by estimating the mean number of structure items observed or process items in each health care facility over the past 3 months. We thereafter performed a geospatial analysis by joining the geospatial coordinate data for each facility with the mean quality score for each facility. A sub-national analysis of obstetric care quality and change in quality over time was conducted and illustrated using choropleth maps. The maps were based on the official administrative boundary derived from GADM.

\section{Results}

In 2013, a total of $\mathrm{N}=907$ facilities were surveyed. With regards to facility location, less than half $(42.7 \%)$ of the facilities located in urban area had normal delivery services available, similarly less than half (43.2\%) of those in rural areas had normal delivery service. Just over 
one-third (37.6\%) of primary care facilities had delivery services present, in contrast majority (77.7\%) of secondary care facilities had delivery services. More than half (57.0\%) of government/public facilities had normal delivery services present, less than one-third of NGO and private facilities (28.3\% and 30.4\% respectively) had normal delivery services. Less than half $(47.4 \%)$ of facilities with laboratory services had normal delivery services. (See Table 1$)$. In 2017, a total of $N=1,033$ facilities were surveyed. In terms of facility location, just over one-third (38.4\%) of the facilities located in urban area had normal delivery services available, similarly only about one-third $(34.3 \%)$ of those in rural areas had normal delivery service. Less than one-third (28.8\%) of primary care facilities had delivery services present, in contrast majority $(83.2 \%)$ of secondary care facilities had delivery services. Less than half $(45.3 \%)$ of government/public facilities had normal delivery services present, less than onethird of NGO and private facilities (29.9\% and $29.3 \%$ respectively) had normal delivery services. Less than half (45.2\%) of facilities with laboratory services had normal delivery services. (See Table 1).

\footnotetext{
[Insert Table 1]

Table 2 shows the BEmOC and CEmOC quality index in 2013 and 2017 at primary and secondary care facilities. A higher proportion of secondary care facilities had basic infrastructures and equipment when compared to primary care facilities. However, some basic structure items were lacking in most facilities.

In 2013 , only $2.7 \%$ and $17 \%$ of primary and secondary facilities respectively were connected to the electricity grid. Only a few facilities had vacuum extractor [primary facilities $(4.1 \%)$; Secondary facilities (28.7\%)]. Vacuum aspirators, dilation and curettage kits were also available in only a few facilities [primary facilities (14.6\%); Secondary facilities (35.1\%)]. Only $14.6 \%$ of primary facilities used partographs routinely, while one-third (34\%) of secondary care facilities routinely used partographs. Medical mask was available in only 37.6 $\%$ of primary care facilities and in just over half (53.2\%) of secondary facilities. Medical gowns and suture materials such as needles, scissors or blades were available in most primary and secondary facilities. Regarding the availability of essential medications, less than half (47.1\%) of primary care facilities had injectable uretronic (oxytocin), only 19\% of primary
} 
facilities had injectable diazepam. In contrast, over three quarter $(77.7 \%)$ of secondary care facilities had injectable uretronic (oxytocin), less than half (48.9\%) of secondary care facilities had injectable diazepam. In terms of newborn care, resuscitation bags, mask or tube were available in only $23.4 \%$ and $75.5 \%$ of primary care facilities and secondary care facilities respectively, incubators were available in $6.8 \%$ and $29.8 \%$ of primary care and secondary care facilities respectively.

Regarding basic emergency obstetric care, primary facilities had performed less BEmOC related services compared to secondary facilities during the preceding 3 months. Only $44.7 \%$ of primary facilities had administered parenteral antibiotics, compared to $90.4 \%$ of secondary care facilities. Just $18.0 \%$ of primary care facilities had administered parenteral anticonvulsants in the past 3 months preceding the audit. In contrast $71.3 \%$ of secondary facilities have administered parenteral anticonvulsant during the same time frame.

Comprehensive emergency obstetric care services were not routinely performed in majority of primary care facilities, with only $1 \%$ performing blood transfusion; in contrast, just less than half of secondary facilities $(47.9 \%)$ had performed blood transfusion during the same time frame. Only $2.7 \%$ of primary care facilities provided Caesarean section in the past 3 months, whereas over three-quarter $(77.9 \%)$ of secondary care facilities had performed caesarean surgery in the past 3 months.

In 2017, there was a modest improvement in many of the indicators when compared to 2013. Some basic infrastructures such as electricity and other basic equipment were still widely lacking. Only a few health facilities were connected to the electricity grid [primary facilities (3.6\%); Secondary facilities $(6.5 \%)]$. Similarly, very few facilities had a manual vacuum extractor [primary facilities (8.3\%); Secondary facilities (27.5\%)]. Vacuum aspirators, dilation and curettage kits were equally only available in few facilities [primary facilities $(23 \%)$; Secondary facilities $(38.5 \%)]$. Just over a third of both primary and secondary facilities routinely used partographs (33.7\% and 37.6\% respectively). Medical mask was available in $61.6 \%$ and $67.1 \%$ of primary and secondary facilities respectively. Medical gowns and suture materials such as needles, scissors or blades were available in most primary and secondary facilities. Regarding the availability of essential medications, about two-third (67.1\%) of primary care facilities had injectable uretronics (oxytocin), majority (80.7\%) of secondary facilities had injectable uretronics. Only $24.6 \%$ and $45.9 \%$ of primary facilities and secondary facilities respectively had injectable diazepam. In terms of newborn care, 
resuscitation bags, mask or tube were available in only $46.8 \%$ and $81.7 \%$ of primary care facilities and secondary care facilities respectively, incubators were available in $9.1 \%$ and $33 \%$ of primary care and secondary care facilities respectively.

Regarding basic emergency obstetric care, a higher proportion of secondary facilities had performed $\mathrm{BEmOC}$ related indicators over the preceding 3 months compared to primary facilities. Only $27.4 \% \%$ of primary facilities had administered parenteral anticonvulsants, compared to $68.8 \%$ of secondary care facilities over the past 3 months. Similarly, $46.8 \%$ of primary care facilities had performed removal of retained products in the 3 months preceding the audit, compared to $65.1 \%$ of secondary care facilities.

Comprehensive emergency obstetric care services were much less performed in primary care facilities, with only $5.6 \%$ performing blood transfusion; in contrast, just less than half of secondary facilities $(49.5 \%)$ have performed blood transfusion during the same time frame. Only $5.6 \%$ of primary care facilities provided Caesarean section during this period, whereas nearly three-quarters $(74.3 \%)$ of secondary care facilities had performed caesarean section.

\section{[Insert Table 2]}

Table 3 shows a Wilcoxon signed rank statistical test to test for significant difference in quality between 2013 and 2017 for primary and secondary facilities respectively.

Percentage point change for indicators at secondary facilities were mixed, with slight improvement in some indicators and a decline in others. Regarding infrastructure, staffing, delivery pack and running water were the most improved indicator at primary care facilities $(14.7 \%, 24.1 \% \& 21 \%$ respectively). The most improved infrastructure at secondary care facilities include: running water, staffing, tubing's and connectors for endotracheal tube $(10.3 \%, 8.3 \%$ and $5.5 \%$ respectively). Basic structure items such as electricity connection and speculum became less available at secondary facilities in 2017, compared to $2013(-10.5 \%$ and $-7.3 \%$ respectively).

In terms of process indicators, there was improvement in primary care facilities, whereas availability either declined or slightly increased at secondary care facilities. Although only a very small number of primary care facilities conducted CEmOC related services in 2013 and 
2017, however there was a slight increase during this period: blood transfusion (4.6\%) and caesarean section $(2.9 \%)$. Secondary care facilities had a decline in CEmOC: caesarean section (-3.4).

Overall, Wilcoxon signed rank test showed that primary care facilities witnessed significant improvements in indicators of structure $(p<0.001)$ and BEmOC $(p=0.03)$ between 2013 and 2017. There was no significant change in quality of CEmOC in primary facilities ( $p$ $=0.18)$. There was no significant change in any of the structure or process indicators at secondary care facilities (see Table 3 ).

[Insert Table 3]

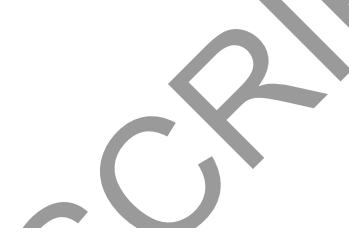

Overall, Ouest and Nord-Ouest departments have the highest concentration of primary care facilities. In 2013, primary care facilities on the Gonave Island, Saint-Louis-du-Nord arrondissement, Cap-Haïtien, Dessalines arrondissement, and Corail arrondissement and some parts of Ouest department had the lowest mean quality. While facilities in Cerca-laSource in Center department, certain parts of Ouest department, parts of Grand'Anse Department and Saint-Louis-du-sud had the best mean quality.

\section{[Insert Figure 1]}

The quality of care improved significantly between 2013 and 2017 in many primary care facilities. In 2017, facilities in Gonave Island, Port-de-paix arrondissement, Jacmel, Les Anglais, Torbeck commune had the lowest mean quality (See Figure 1).

\section{[Insert Figure 2]}

In 2013, the quality of secondary health facilities was mixed with some between (0.5-0.6) and others above average. Secondary facilities in Gros-Morne Arrondissement, Cerca-la-Source, Thiotte commune and Les-Anglais commune had the lowest mean quality, while facilities in some parts of Artibonite, Centre department and part of Ouest were of the highest quality.

In 2017, the quality of secondary facilities remained mostly unchanged, with slight decline in some regions compared to 2013. Facilities with the lowest quality were in parts of Nord Ouest department, Gros-Morne Arrondissement, Cerca-la-Source, Thiotte and Les-Anglais 
communes. The facilities with highest quality remained concentrated around Centre and Artibonite departments (See Figure 2).

\section{[Insert Figure 3]}

Analysis of change in obstetric care quality in primary and secondary facilities between 2013 and 2017 shows that primary care facilities made the most significant improvement, while most secondary facilities declined, with only a few improving in quality.

Primary facilities located around Ouest, Nord Ouest and Sud departments did not improve in quality, while those in Artibonite and parts of Centre department as well as in Cap-Haitien and Pestel communes made the most significant improvement.

Many secondary facilities declined in quality or made no significant improyement, except very few facilities in Nord Ouest, Centre and Sud which made a slight improvement (see Figure 3).

\section{Discussion}

\section{Statement of principal findings}

We have conducted a cross-sectional study exploring the quality of emergency obstetric care services available at Haitian health care facilities at two time-points (2013 and 2017). We found that the quality of obstetric care services available at primary care facilities were lower compared to those of secondary care facilities. This finding is consistent with previous studies (27). Kruk et al. assessed the quality of basic maternal care functions at health facilities in five African countries, and found that mean quality of care was about two-fold higher in secondary facilities compared to primary facilities (Mean(SD): 0.38(0.19) Versus $0.77(0.14)(3)$

Furthermore, our study revealed that primary care facilities made significant improvement in quality during the five years period, whereas obstetric care quality in many secondary facilities either declined or remained the same. Sub-national analysis showed that a higher number of healthcare facilities are concentrated around Port-au-Prince arrondissement and other parts of Ouest department; this pattern of distribution has been corroborated by other studies conducted in Haiti (28). 
Our analysis showed that despite the higher proportion of facilities concentrated in this region, the quality of some of these facilities were low. In contrast, Centre department has lesser concentration of health facilities, however the quality of the facilities in this area was relatively higher. Similarly, the quality of facilities in Nord Ouest were also among the lowest. The findings in this region is in line with a previous study investigating the quality of emergency health care at all facilities in North East Haiti (29).

We identified basic structure items such as connection to the electricity grid as a major problem across most facilities in Haiti, with little improvement over the five-year period. It is however notable that while the issue of electricity was highlighted in another study conducted in Haiti, the study mentioned that most health facilities relied on alternative electricity source from electric generators (29). We found that while basic structure items such as water improved at both primary and secondary facilities, basic equipment such as vacuum aspirators and vacuum extractors were widely unavailable at many facilities; this may be linked to the lack of electricity to run this equipment.

Comprehensive obstetric care was widely unavailable at primary care facilities, as shown by indicators such as blood transfusions and caesarean sections provided over the past 3 months; this trend has been shown in other LMIC (3). This trend underscores the need to prioritise effective referrals in primary facilities: by ensuring timely referral, optimal emergency transport, and good roads amongst other factors $(3,14,15)$. Other authors have highlighted in their work that even though services such as blood transfusion and surgical care are considered part of comprehensive Emergency Obstetric Care (CEmOC), and are not required in every delivery, women must readily have access to them when complications arise (7).

\section{Interpretation within the context of the wider literature}

By conducting a sub-national analysis, we were able to identify the administrative regions with facilities most in need of quality improvement. It has been shown that the minimum acceptable level of obstetric care is four basic and one comprehensive emergency obstetric care facility per 500,000 women $(8,9)$. All women with direct obstetric complications are to be treated in emergency obstetric care facilities $(8,9)$. Our finding can be further enhanced by linking the population estimates in different communities to the quality of obstetric care available. 
The quality of obstetric care service has also been shown to affect utilisation decision. A study conducted by Wang et al., showed that infrastructure quality at health care facilities in Haiti was directly associated with the utilisation of obstetric care services by members of the community (11). Contrarily, Gage et al., showed in their work that utilisation of facilities for childbirth was not associated with the service delivery quality, although they found that it was associated with utilisation of other services such as Antenatal care (25).

Our study showed that primary facilities made significant progress in quality improvement. This finding is a positive trend; hence policy makers and stakeholders must develop measures to consolidate on this trend for a number of reasons. Firstly, majority of obstetric care facilities in Haiti are primary care facilities, secondly majority of deliveries still take place in primary care facilities, to maximise access, the World Health Organization in partnership with policy makers in LMIC promotes the "close- to-client" firstlevel maternal and newborn care approach to the provision of delivery and newborn care, wherein delivery for most women occur in primary care facilities and complications are referred to higher level facilities $(7,32)$. Unfortunately, we found that secondary facilities made no progress in quality improvement. This is a negative trend, and this may easily hold back the overall progress in obstetric service quality improvement in Haiti, since the secondary facilities are supposed to serve as an important hub for referrals

In assessing the quality of emergency obstetric care in Haiti, we utilised the signal functions of emergency obstetric care indicators. In addition, a proxy of the structure, process and outcome model developed in the work of Donabedian, was used to explore quality (20). Further insights from Donabedian's work reveals that improving the quality of obstetric care in health systems settings such as in Haiti may require a more robust and comprehensive approach, one that goes beyond simply improving basic infrastructure and processes. Other factors such as the cost of care, community preference, provider-client relationship, fair and equitable distribution of care needs to be closely addressed (21).

\section{Strengths and limitations}

The study has some strengths, most importantly, to the best of our knowledge, it represents the first attempt to explore changes in quality of obstetric care over time in a LMIC. In addition, the data we have utilised is robust, and is representative of all health facilities in Haiti. 
There are a few limitations to our study. Firstly, our findings should be interpreted with caution, because each survey is a snapshot of EmOC in the most recent three months preceding the survey; annual reclassification has been recommended when measuring obstetric care status over time, for programmatic reasons (9). We only assessed change in the quality of facility infrastructure and availability of services. Measuring changes in service delivery through the analysis of indicators such as wait times, clients' perception, or care experience, was not within the scope of our study, however the importance of these service indicators have been highlighted elsewhere (25).

\section{Implications for policy, practice, and research}

Our findings have several implications for quality of obstetric care in Haiti. Firstly, measuring changes in indicators of emergency obstetric care services have been shown to be an important proxy in assessing progress in the reduction of maternal mortality (8). Hence, government authorities, policy makers, and other donor agencies can target resources more effectively, using the quality trends and patterns that we have enumerated. Secondly, some facilities, especially primary care facilities in certain administrative regions, have shown significant improvement in quality of care, It is important to identify facilities with optimal standards and the most improved facilities and to use them as a model or template for improvement elsewhere in Haiti. The need to improve healthcare quality using this approach, has also been highlighted in another study conducted in Haiti (28).

\section{Conclusion}

Our study can assist Haitian health authorities, program planners, donor agencies and policy makers in identifying areas of the country where obstetric care services require improvement and strengthening. This study may also serve as a guide for monitoring sub-national trends and change over time in the quality of obstetric care in other LMIC.

\section{Contributorship}

AEA conceptualised the study, developed the study design and protocol, extracted the data, contributed to the data analysis, and drafted the manuscript. JW contributed to the study protocol and conducted the Geospatial analysis. JS contributed to the study design and proofread the article.

\section{Ethics and other permissions}


Permission to use the SPA survey data was granted by Measure DHS. Ethics approval exemption was granted for the analysis of the secondary data by the authors' institution.

\section{Funding}

No funding was received for this project.

\section{Conflict of interest statement}

None declared.

\section{Acknowledgements}

The authors would like to acknowledge the contributions of Professor Hong Zhou and the team at UNICEF China Office.

\section{Data availability}

The data is available upon reasonable request and permission from the Demographic and Health survey program. (https://dhsprogram.com/Data/) 


\section{References}

1. Do M, Wang W, Hembling J, Ametepi P. Quality of antenatal care and client satisfaction in Kenya and Namibia. Int J QualHeal Care [Internet]. 2017 Apr 1 [cited 2020 Oct 22];29(2):183-93. Available from:

https://academic.oup.com/intqhc/article/29/2/183/2966271

2. Kruk ME, Gage AD, Arsenault C, Jordan K, Leslie HH, Roder-DeWan S, et al. Highquality health systems in the Sustainable Development Goals era: time for a revolution. Lancet Glob Heal [Internet]. 2017 Nov 1;6(11):e1196-252. Available from: https://doi.org/10.1016/S2214-109X(18)30386-3

3. Kruk ME, Leslie HH, Verguet S, Mbaruku GM, Adanu RMK, Langer A. Quality of basic maternal care functions in health facilities of five African countries: an analysis of national health system surveys. Lancet Glob Heal [Internet]. 2016 Nov 1 [cited 2020 Aug 24];4(11):e845-55. Available from: www.thelancet.com/lancetgh

4. Kruk ME, Gage AD, Joseph NT, Danaei G, García-Saisó S, Salomon JA. Mortality due to low-quality health systems in the universal health coverage era: a systematic analysis of amenable deaths in 137 countries. Lancet [Internet]. 2017 Nov 17 [cited 2020 Oct 22];392(10160):2203-12. Available from: http://dx.doi.org/10.1016/

5. Thaddeus S, Maine D. Too To Walk : Maternal Mortality in. Soc Sci Med. 
1994;38(8):1091-110.

6. Carvalho Pacagnella R, Guilherme Cecatti J, Jose Osis M, Paulo Souza J.

Reproductive Health Matters An international journal on sexual and reproductive health and rights The role of delays in severe maternal morbidity and mortality: expanding the conceptual framework. Reprod Health Matters [Internet]. 2012 Jun [cited 2021 May 9];20(39):155-63. Available from: https://www.tandfonline.com/action/journalInformation?journalCode=zrhm21

7. Gage AD, Carnes F, Blossom J, Aluvaala J, Amatya A, Mahat K, et al. In Low- And Middle-Income Countries, Is Delivery In High- Quality Obstetric Facilities Geographically Feasible? 2021;

8. Maine D, Wardlaw TM, Ward VM, McCarthy J, Birnbaum A, Akalin MZ, et al. Guidelines for Monitoring the Availability and Use of Obstetric Services. Geographical. 1997. 1-103 p.

9. McCarthy A. Monitoring Emergency Obstetric Care. J Obstet Gynaecol (Lahore). 2010;30(4):430.

10. Leslie HH, Spiegelman D, Zhou X, Kruk ME. Service readiness of health facilities in and the United Republic of Tanzania. Bull World Heal Organ. 2017;

11. Wang W, Winner M, Burgert C, Colston J. Influence of Service Readiness on Use of Facility Delivery Care: A Study Linking Health Facility Data and Population Data in Haiti. 2014.

12. Sheffel A, Zeger S, Heidkamp R, Munos MK. Development of summary indices of antenatal care service quality in Haiti, Malawi and Tanzania. BMJ Open [Internet]. 2019 Dec 2 [cited 2020 Aug 20];9(12):e032558. Available from:

\section{http://bmjopen.bmj.com/}

13. Hulton LA, Matthews Z, Stones RW. Applying a framework for assessing the quality of maternal health services in urban India. Soc Sci Med. 2007 May 1;64(10):2083-95.

14. Banke-Thomas A, Wright K, Sonoiki O, Banke-Thomas O, Ajayi B, Ilozumba O, et al. Assessing emergency obstetric care provision in low- and middle-income countries: a systematic review of the application of global guidelines [Internet]. Vol. 9, Global Health Action. Taylor and Francis Ltd.; 2016 [cited 2021 May 11]. p. 31880. Available 
from: https://doi.org/10.3402/gha.v9.31880

15. Bosomprah S, Tatem AJ, Dotse-Gborgbortsi W, Aboagye P, Matthews Z. Spatial distribution of emergency obstetric and newborn care services in Ghana: Using the evidence to plan interventions. Int J Gynecol Obstet [Internet]. 2016 Jan 1 [cited 2021 May 11];132(1):130-4. Available from: http://dx.doi.org/10.1016/j.ijgo.2015.11.004

16. Gabrysch S, Simushi V, Campbell OMR. Availability and distribution of, and geographic access to emergency obstetric care in Zambia. Int J Gynecol Obstet. 2011 Aug 1;114(2):174-9.

17. Hirose A, Borchert M, Cox J, Alkozai AS, Filippi V. Determinants of delays in travelling to an emergency obstetric care facility in Herat, Afghanistan: An analysis of cross-sectional survey data and spatial modelling. BMC Pregnancy Childbirth [Internet]. 2015 Feb 5 [cited 2021 May 11];15(1):1-13. Available from: http://www.aims.org.af/

18. Duysburgh E, Zhang WH, Ye M, Williams A, Massawe S, Sié A, et al. Quality of antenatal and childbirth care in selected rural health facilities in Burkina Faso, Ghana and Tanzania: Similar finding. Trop Med Int Heal [Internet]. 2013 May 1 [cited 2021 May 11];18(5):534-47. Available from: https://onlinelibrary.wiley.com/doi/full/10.1111/tmi.12076

19. Nesbitt RC, Lohela TJ, Manu A, Vesel L, Okyere E, Edmond K, et al. Quality along the continuum: A health facility assessment of intrapartum and postnatal care in Ghana. PLoS One [Internet]. 2013 Nov 27 [cited 2021 May 11];8(11):81089. Available from: www.plosone.org

20. Donabedian A. The quality of care. How can it be assessed? JAMA. 1988 Sep;260(12):1743-8.

21. Donabedian A. Donabedian 1990.pdf. Vol. 114, Archives of Pathology \& Laboratory Medicine. 1990. p. 1115-8.

22. Demographic and Health Surveys (DHS) program. The DHS Program - Service Provision Assessments (SPA) [Internet]. [cited 2020 Oct 22]. Available from: https://dhsprogram.com/What-We-Do/Survey-Types/SPA.cfm

23. Mirkovic KR, Lathrop E, Hulland EN, Jean-Louis R, Lauture D, D'Alexis GD, et al. 
Quality and uptake of antenatal and postnatal care in Haiti. BMC Pregnancy Childbirth [Internet]. 2017 Feb 2 [cited 2021 May 27];17(1):1-10. Available from: https://link.springer.com/articles/10.1186/s12884-016-1202-7

24. Kolbe AR, Hutson RA, Shannon H, Trzcinski E, Miles B, Levitz N, et al. Mortality, crime and access to basic needs before and after the haiti earthquake: A random survey of port-au-prince households. Med Confl Surviv [Internet]. 2010 Oct [cited 2021 May 28];26(4):281-97. Available from: https://pubmed.ncbi.nlm.nih.gov/21314081

25. Gage AD, Leslie HH, Bitton A, Jerome JG, Joseph JP, Thermidor R, et al. Does quality influence utilization of primary health care? Evidence from Haiti. Global Health [Internet]. 2017 Jun 20 [cited 2020 Dec 14];14(1):59. Available from: https:/globalizationandhealth.biomedcentral.com/articles/10.1186/s12992-018-0379-0

26. Watts J. Haiti making good progress in health but challenges remain [Internet]. Vol. 384, The Lancet. Lancet Publishing Group; 2014 [cited 2021 May 28]. p. 1413-4. Available from: http://dx.doi.org/10.1016/

27. Kruk ME, Leslie HH, Verguet S, Mbaruku GM, Adanu RMK, Langer A. Quality of basic maternal care functions in health facilities of five African countries: an analysis of national health system surveys. Lancet Glob Heal. 2016 Nov 1;4(11):e845-55.

28. Gage AD, Leslie HH, Bitton A, Jerome JG, Thermidor R, Joseph JP, et al. Évaluation de la qualité des soins de santé primaires en Haïti. Bull World Health Organ [Internet]. 2017 Mar 1 [cited 2020 Dec 14];95(3):182-90. Available from: /pmc/articles/PMC5328114/?report=abstract

29. De Wulf A, Aluisio AR, Muhlfelder D, Bloem C. Emergency Care Capabilities in North East Haiti: A Cross-sectional Observational Study. Prehosp Disaster Med [Internet]. 2015 Oct 19 [cited 2020 Dec 13];30(6):553-9. Available from:

https://www.cambridge.org/core/journals/prehospital-and-disastermedicine/article/abs/emergency-care-capabilities-in-north-east-haiti-a-crosssectionalobservational-study/41A1CFE0351036C87F753FC3AB0DCAFA

30. Hirose A, Borchert M, Cox J, Alkozai AS, Filippi V. Determinants of delays in travelling to an emergency obstetric care facility in Herat, Afghanistan: An analysis of cross-sectional survey data and spatial modelling. BMC Pregnancy Childbirth [Internet]. 2015 Feb 5 [cited 2020 Dec 14];15(1):14. Available from: 
http://bmcpregnancychildbirth.biomedcentral.com/articles/10.1186/s12884-015-04351

31. Assarag B, Dujardin B, Delamou A, Meski FZ, De Brouwere V. Determinants of maternal near-miss in morocco: Too late, too far, too sloppy? PLoS One [Internet]. 2015 Jan 22 [cited 2020 Dec 14];10(1). Available from: /pmc/articles/PMC4303272/?report=abstract

32. World Health Organization. attending to 136 million births, every year RISKING DEATH TO GIVE LIFE. 2005.

Table 1: Characteristic of health care facilities in Haiti in 2013 and 2017

20132013
2017

Delivery services present No delivery service

$n(\%)$

Facility location

Urban $151(42.7)$ $238(43.2)$

Rural

$95(37.6)$

$94(77.7)$

Management type

Government/Public 195(57.0)

NGO/not for profit

$47(28.3)$

Private/profit

$65(30.4)$

Faith-based/Mixed

$82(44.8)$

Laboratory services

$\begin{array}{ll}\text { Available } & 312(47.4) \\ \text { Not available } & 75(30.9)\end{array}$

$489(62.4)$

$27(22.3)$

147(43.0)

119(71.7)

149(69.6)

101(55.2)

$346(52.6)$

$168(69.1)$
Delivery services present No delivery services

$n(\%)$ $\mathrm{n}(\%)$
233(61.6)

413(65.7)

624(71.2)

22(16.8)

188(54.7)

122(70.1)

52(29.9)

88(29.3)

212(70.7)

124(65.6)
$352(54.8)$

646(64.2) 
Table 2: Quality of basic and comprehensive emergency obstetric care at primary and secondary care facilities providing normal delivery care.

Indicators of structure

Schedule $24 \mathrm{~h}$ staffing observed

Connected to electricity grid

Running water observed

Sharps container observed

Skin disinfectant

Delivery bed

Delivery pack

Cord clamp

Speculum

Episiotomy scissors

Suture material with needle

Manual vacuum extractor

Vacuum aspirator or D\&C kit

Anaesthesia giving set observed functioning

Tubings and connectors for endotracheal tube

Partograph availability and routine use

Single use syringes

Medical Mask

Medical gowns

Suture material with needle

Sterile scissors or blade

Injectable uretronic (oxytocin)

Injectable diazepam

Injectable magnesium sulfate

Injectable antibiotic (ceftriaxone)

Infant resuscitation bag/mask or tube

Incubator

\section{Indicators of process of BEmOC}

Assisted vaginal delivery past 3 months

Parenteral antibiotics administered past 3 mnths

67.5

44.7

Parenteral oxytocic drugs administered past 3 mnths 58.6

Use of parenteral anticonvulsant past 3 months

18.0

Removal of retained product past 3 months

40.7

Manual removal of placenta

44.4

1.0

2.7

Indicators of process of CEmOC

Blood transfusion in the past 3 months

Caesarean section provided past 3 months
73.6

17.0

85.1

93.6

85.1

97.9

88.3

93.6

92.6

90.4

90.4

28.7

35.1

83.3

87.2

34.0

86.2

53.2

96.8

90.4

90.4

77.7

48.9

72.3

47.9

75.5

29.8

94.7

90.4

98.9

71.3

72.3

70.2

47.9

77.7

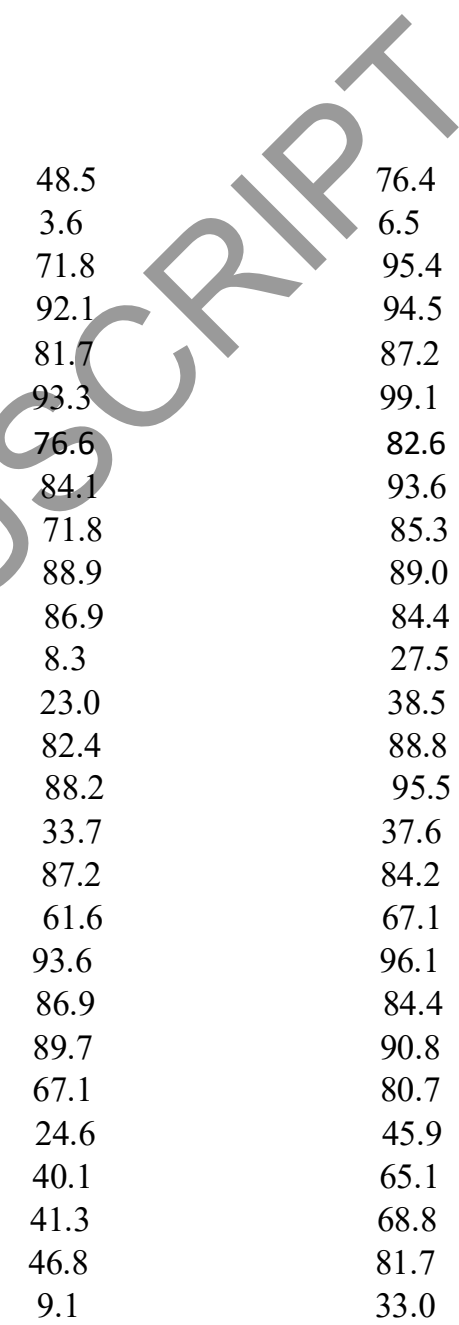

80.2

98.2

96.3

97.5

68.8

65.1

73.4

In $2013(N=389) ; \operatorname{In} 2017(N=361)$ 
Figure 1: Sub-national analysis of mean obstetric care quality in Haitian primary facilities

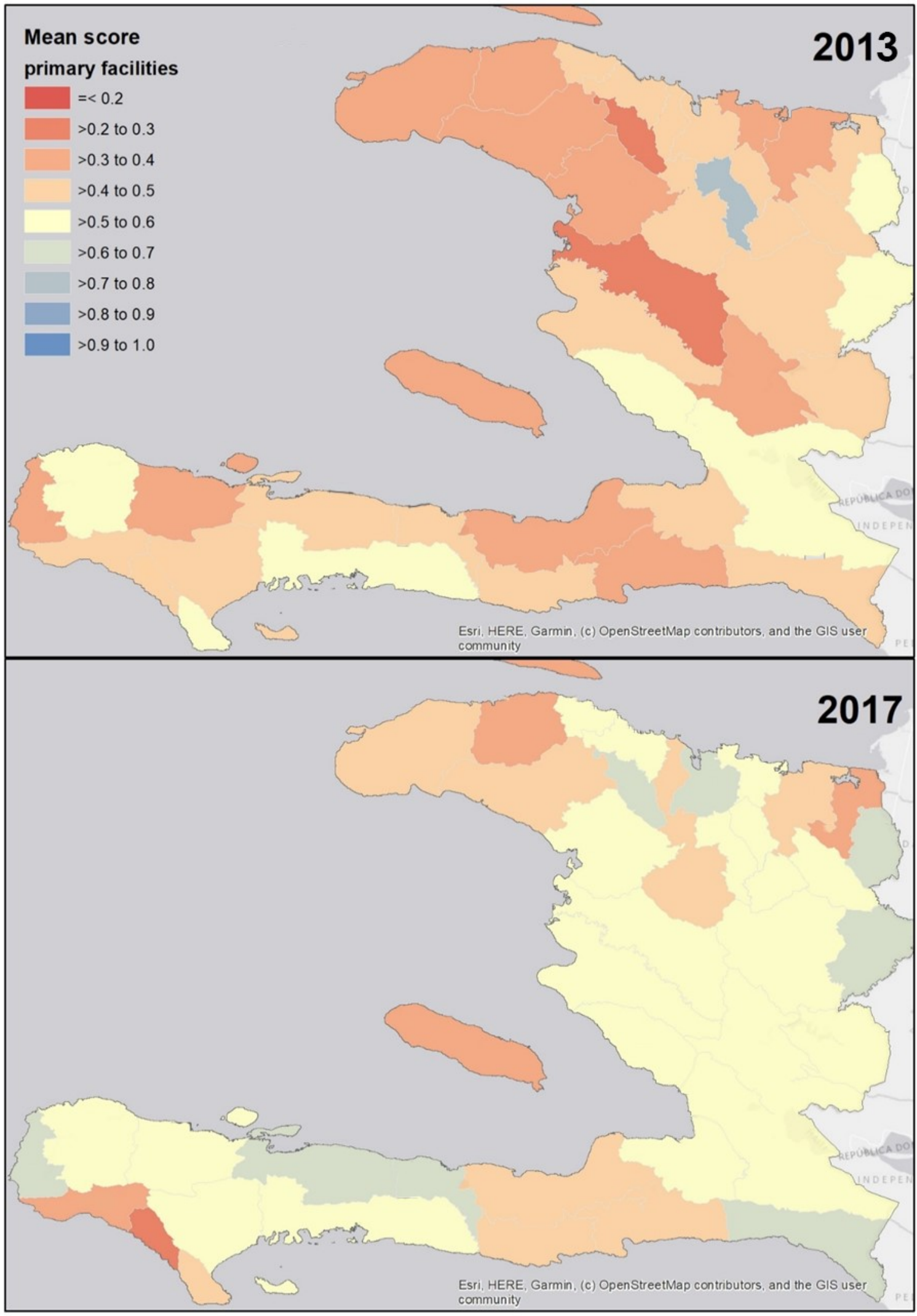


Figure 2: Sub-national analysis of mean obstetric care quality in Haitian secondary facilities.

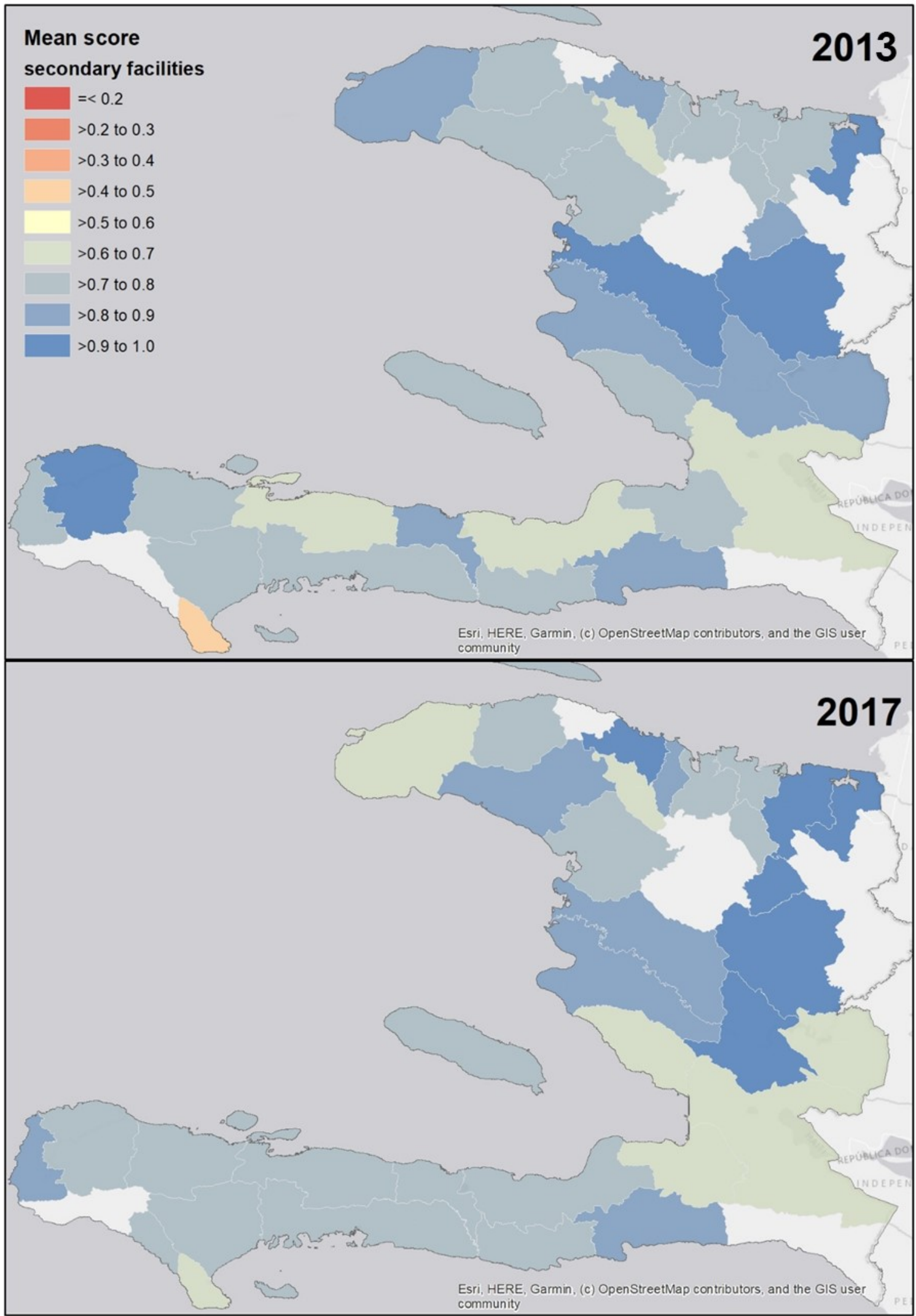


Figure 3

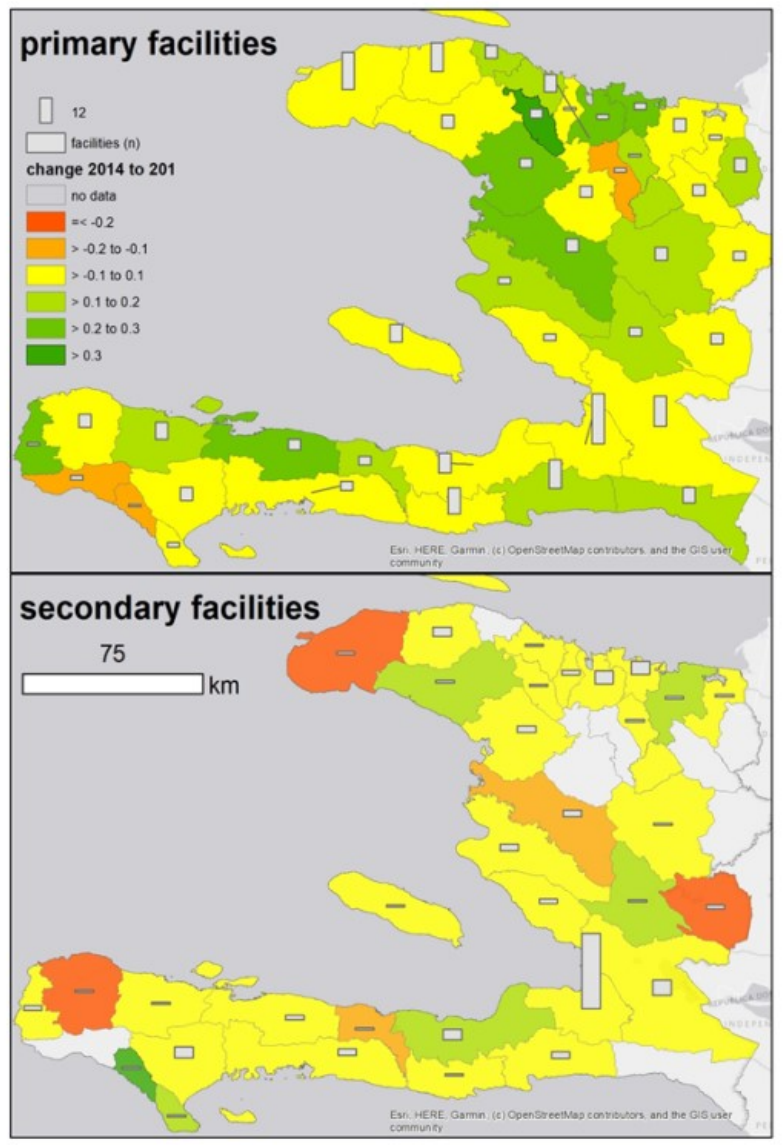

Figure 3: Change in mean facility quality betw een 2014 and 2017

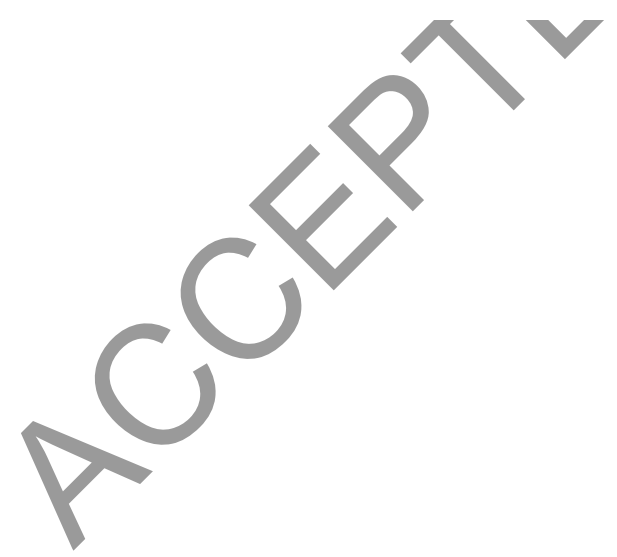

